\title{
An autonomous adaptive low-power instrument platform (AAL-PIP) for remote high-latitude geospace data collection
}

\author{
C. R. Clauer ${ }^{1,5}$, H. Kim ${ }^{1,5}$, K. Deshpande ${ }^{1,5}$, Z. Xu ${ }^{1,5}$, D. Weimer ${ }^{1,5}$, S. Musko ${ }^{2}$, G. Crowley ${ }^{3}$, C. Fish ${ }^{4}$, R. Nealy ${ }^{5}$, \\ T. E. Humphreys ${ }^{6}$, J. A. Bhatti ${ }^{6}$, and A. J. Ridley ${ }^{2}$ \\ ${ }^{1}$ Center for Space Science Engineering and Research, Virginia Polytechnic Institute and State University, \\ Blacksburg, VA, USA \\ ${ }^{2}$ Space Physics Research Laboratory, University of Michigan, Ann Arbor, MI, USA \\ ${ }^{3}$ Atmospheric \& Space Technology Research Associates, Boulder, CO, USA \\ ${ }^{4}$ Space Dynamics Laboratory, Utah State University, Logan, Utah, USA \\ ${ }^{5}$ Bradley Department of Electrical and Computer Engineering, Virginia Polytechnic Institute and State University, \\ Blacksburg, VA, USA \\ ${ }^{6}$ Department of Aerospace Engineering and Engineering Mechanics, The University of Texas at Austin, Austin, TX, USA
}

Correspondence to: C. R. Clauer (rclauer@vt.edu)

Received: 28 April 2014 - Published in Geosci. Instrum. Method. Data Syst. Discuss.: 16 June 2014

Revised: 28 August 2014 - Accepted: 11 September 2014 - Published: 10 October 2014

\begin{abstract}
We present the development considerations and design for ground-based instrumentation that is being deployed on the East Antarctic Plateau along a $40^{\circ}$ magnetic meridian chain to investigate interhemispheric magnetically conjugate geomagnetic coupling and other space-weatherrelated phenomena. The stations are magnetically conjugate to geomagnetic stations along the west coast of Greenland. The autonomous adaptive low-power instrument platforms being deployed in the Antarctic are designed to operate unattended in remote locations for at least 5 years. They utilize solar power and AGM storage batteries for power, two-way Iridium satellite communication for data acquisition and program/operation modification, support fluxgate and induction magnetometers as well as a dual-frequency GPS receiver and a high-frequency (HF) radio experiment. Size and weight considerations are considered to enable deployment by a small team using small aircraft. Considerable experience has been gained in the development and deployment of remote polar instrumentation that is reflected in the present generation of instrumentation discussed here. We conclude with the lessons learned from our experience in the design, deployment and operation of remote polar instrumentation.
\end{abstract}

\section{Introduction}

Improvement in the quality and resolution of data for use in scientific analysis and discovery is a major driver in the advancement of geophysical science. Improvements are accomplished by increasing the spatial distribution of measurements and by increasing the quality and temporal resolution of the samples. This is particularly true in space science and in the investigation of space weather - the dynamic variation of electrical currents and energetic charged particle populations around the Earth and in the upper atmosphere that are produced by interactions of the supersonic solar wind with the geomagnetic field. The solar wind flows around and distorts the geomagnetic field, forming the magnetospheric cavity in the flow. The magnetic field is compressed on the sunward side, and drawn out into a long comet-like tail on the nightside of the Earth. Various processes couple energy and momentum from the solar wind into the magnetosphere to drive electric currents and energize plasma.

Ground arrays of instruments at high latitudes are particularly advantageous for monitoring space weather phenomena because, due to the dipole nature of the main magnetic field, the entire outer magnetosphere maps to a relatively small region at polar and auroral latitudes in both hemispheres. Measurements by arrays of instruments in the polar regions 
can be used to provide context to better understand observations from satellites in space. Measurements from polar instruments are also vital to the validation of global numerical models that may be used to describe and forecast space weather phenomena. It is, therefore, increasingly important to deploy arrays of geophysical instruments in polar regions to advance our understanding of the complex electrodynamic interactions that comprise space weather. The examination of simultaneous data from both the northern and southern polar regions is also very important because of the considerable asymmetries between the two hemispheres. For example, solar illumination differences between the summer and winter hemisphere produce large asymmetries in the conductance in the two polar ionospheres. The magnetic field in the Southern Hemisphere is significantly weaker and therefore experiences larger amounts of energetic particle precipitation into the ionosphere, producing localized channels of higher conductivity.

While the Northern Hemisphere is relatively well instrumented, the southern polar region is not, primarily because of the extreme Antarctic climate and lack of manned facilities with infrastructure to support instrumentation. Due to the lack of manned infrastructure, measurements from the southern polar region have historically been very sparse. However, during the past decade, technical development has enabled the initiation of robust measurement programs using autonomous instrument platforms that can be deployed remotely and operate unattended for extended periods of time. Examples of such systems include the low-power magnetometer (LPM) platforms operated by the British Antarctic Survey (BAS) and the Automated Geophysical Observatory (AGO) program supported by the National Science Foundation (Lessard et al., 2009; Melville et al., 2014). The BAS LPMs are simple yet robust systems that use only solar power and batteries to support the data acquisition system (Kadokura et al., 2008). They store data in solid-state memory and must be visited each year to acquire the data. During these visits, the electronics box with memory and acquisition system are simply swapped with a new system, so the service is simple and rapid. The US AGOs are more complex systems supporting multiple instruments and utilizing both wind and solar power. They also utilize satellite communications (Iridium) to acquire data and monitor the health of the system. Nevertheless, they often require maintenance visits during the summer field season. In the Appendix, we provide Web resources for various autonomous polar system designs taken from the Autonomous Polar Observing Systems Workshop Report .

Another example of remote autonomous system development is the evolving low-power magnetometer platforms developed originally for use on the Greenland ice cap and later for deployment in Antarctica. We report here on the most recent development and deployment of this platform that now supports multiple low-power instruments and is designed to operate unattended for at least 5 years. Designated as an autonomous adaptive low-power instrument platform (AAL-PIP), it is a relatively simple, yet robust, system using only solar power and storage batteries for winter operation. A two-way satellite data link provides data acquisition and system engineering data, and provides the ability to load new software to modify the operation of the system. While any cluster of low-power instruments can be supported, the present systems deployed on the East Antarctic Plateau support (1) a fluxgate magnetometer, (2) an induction magnetometer; (3) the Connected Autonomous Space Environment Sensor (CASES), a new software-defined, dual-frequency Global Positioning System (GPS) receiver; and (4) a highfrequency (HF) radio experiment.

We have a long history of developing and deploying small autonomous instrument systems, beginning in 1990 through the present. A review of this history is given by Musko et al. (2009). Here we will focus our attention on the specific design requirements and changes that have led to the present AAL-PIP. The system is designed for remote deployment by small aircraft onto the Antarctic Plateau and to operate unattended for at least 5 years. Data are stored in solid-state memory but are also transmitted via Iridium satellite telemetry to laboratories at the University of Michigan and Virginia Tech. During the summer, the photo voltaic (PV) panels provide abundant power. During the dark of winter, power is provided only by a bank of lead-acid absorbed glass mat (AGM) batteries. Therefore, during the winter, data are stored in memory and only engineering information is transmitted via the Iridium satellite so that, while using less power we can still monitor the health of the system. Since the Iridium communication link is two-way, it is possible to command the station to send winter data if there is a special event or time interval needed. When the sun comes up again and power becomes abundant, the current data plus the archived data are transmitted.

The CASES dual-frequency GPS receiver requires more power and produces more heat in the electronics box than the other instruments. Therefore, it is not run continuously but rather scheduled to run for specific time intervals. Thus, it does not provide continuous data. The GPS signals are being examined primarily to investigate signal scintillation (variations in amplitude and phase of the signal) with the goal of understanding the associated plasma irregularities that produce the scintillations. The two-way communication with the platforms using the Iridium link enables the capability to modify the operation of the system and schedule the operation of the instruments in different modes as desired. In general, the fluxgate and induction magnetometers are operated in a standard data collection mode, while the GPS and HF radio can be scheduled to operate during specific time intervals, or during specific conditions in the magnetometer data that can be monitored by the system itself, and/or in various modes of operation.

In the event that the system detects a sufficiently low charge state in the batteries during the winter operation, the 


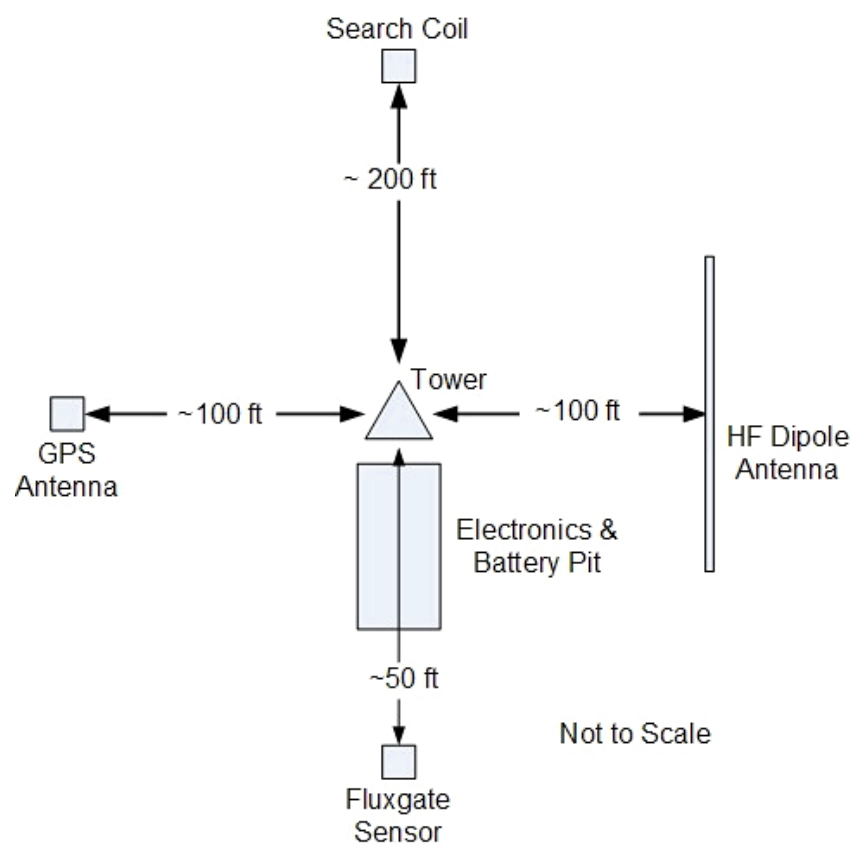

Figure 1. Schematic diagram of the site plan for the deployment of an AAL-PIP system supporting fluxgate magnetometer, search coil (or induction) magnetometer, CASES dual-frequency GPS receiver and HF transceiver experiment.

system will gracefully shut down and wait in this hibernation state until power is detected from the PV panels. When the sun comes up, power from the PV panels is initially used to heat the batteries to a temperature that will permit them to accept charging. The batteries are subsequently charged as the system resumes operation.

We are in the process of manufacturing 4 AAL-PIP systems for remote field deployment on the East Antarctic Plateau to form a chain along the $40^{\circ}$ magnetic meridian. Prior to deployment in the remote location, each station is deployed at the South Pole to test for 1 year. Figure 1 shows a schematic drawing of the deployment plan for an AAL-PIP system. Figure 2 shows an AAL-PIP system during deployment at the South Pole.

\section{Design considerations}

The Antarctic Plateau is an extreme environment, having summer high temperatures that only reach $-14{ }^{\circ} \mathrm{C}$, while the winter lows can reach $-70^{\circ} \mathrm{C}$. The infrastructure on the continent is limited in both availability and accessibility. The South Pole station is one of only a few manned stations that are located within the interior of the continent, and it is open for summer deployment teams only a few months of the year. It is, therefore, necessary to be able to deploy the system within a few days in the field by a small team of two or three people using small aircraft. Since accessing the stations is difficult and costly, once established, each station should be able to operate unattended for several years. Finally, the sun is not available as a power source for a large portion of the year. Thus, our design requirements are that each system must

1. operate unattended at any location on the Antarctic plateau for at least 5 years with no maintenance;

2. instrument power requirements should be low, $\approx 1 \mathrm{~W}$;

3. measure the magnetic field strength with $0.2 \mathrm{nT}$ resolution in three orthogonal components and with $1 \mathrm{~s}$ cadence;

4. measure magnetic field waves in the ultra-lowfrequency (ULF) range $(0.1-5 \mathrm{~Hz})$ with the resolution of tens of $\mathrm{pT} / \sqrt{\mathrm{Hz}}$ over the frequency response in two orthogonal horizontal components;

5. support a newly developed low-cost, software-defined, science-grade, dual-frequency GPS receiver for exploratory scintillation measurements;

6. be able to store at least 1 year of science and engineering data in internal nonvolatile memory;

7. transmit stored and near-real-time data to our laboratories at the University of Michigan and/or Virginia Tech via satellite communication link;

8. time-tag stored data with coordinated universal time (UTC) $\pm 40 \mathrm{~ms}$;

9. include an HF radio experiment to explore radio propagation characteristics between the deployed stations (over ranges of a few hundred to several hundreds of kilometers).

Considerable attention was also devoted to facilitating the deployment of these systems under difficult cold conditions. For example, most of the system can be assembled with gloves on. There are no nuts and bolts to be manipulated and no tools are required. Instead, pins are inserted to secure the tower sections, and T-bolts are used to secure the solar panels to the tower. This is illustrated in Fig. 3, showing a close-up view of the tower assembly with solar panels. The T-bolts secure the solar panels to top and bottom frames, and this assembly is winched to the top of the tower after the tower is raised and secured by guy wires.

Using the specifications described above, the present generation of AAL-PIP was designed as described in the following.

\section{System and thermal design}

The main consideration in the thermal design of the AALPIP is the desire to not have to revisit the site for maintenance. This consideration outweighs the desire for a $100 \%$ 

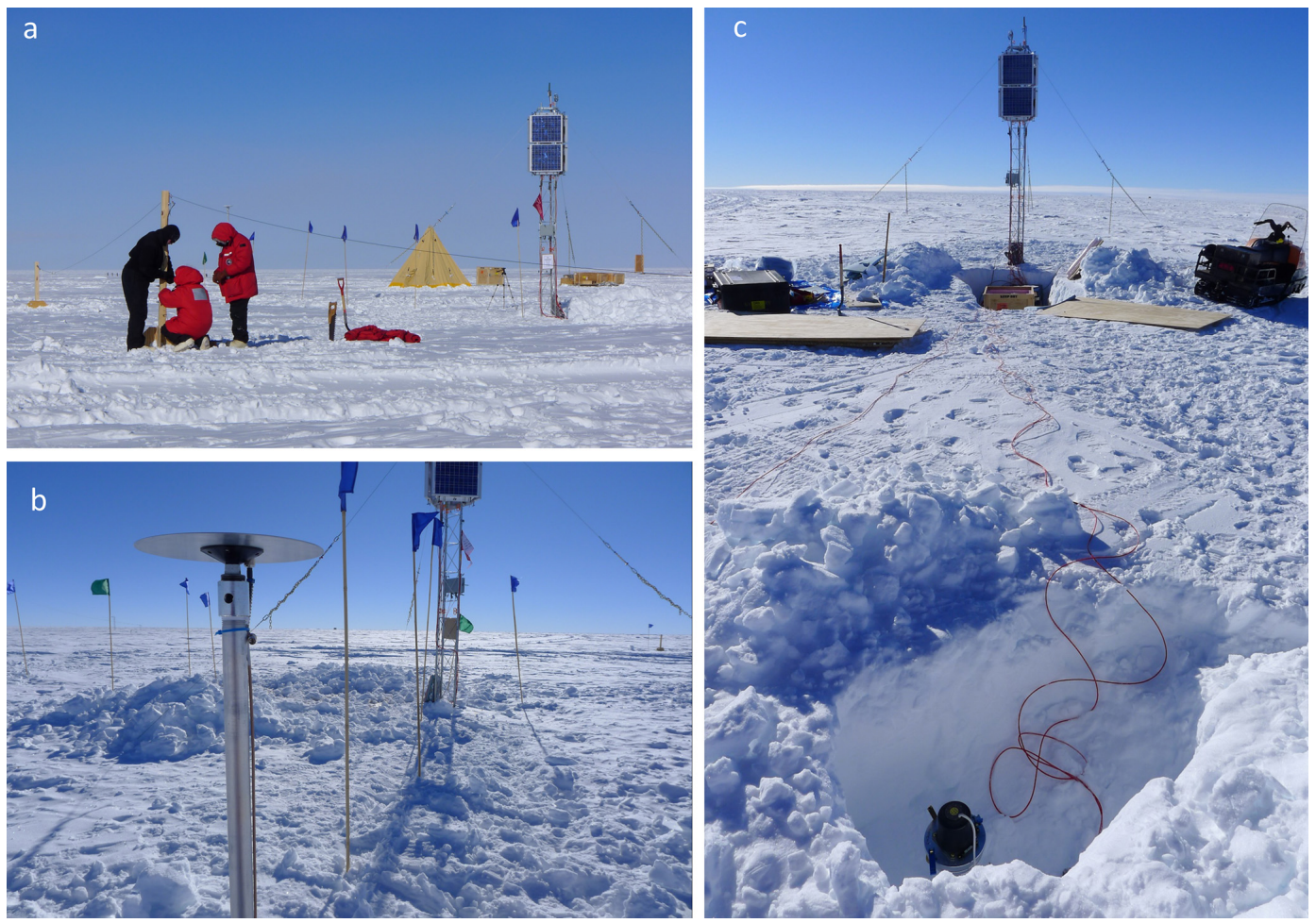

Figure 2. (a) shows HF radio antenna being installed next to system tower. (b) shows the GPS antenna for the dual-frequency CASES receiver mounted on a pole near the tower. (c) shows a view of the installation from the location of the fluxgate sensor pit. At the base of the tower is a pit containing the super-insulated electronics box and the battery box. The tower holds the photo voltaic (PV) panel array as well as antenna for the Iridium satellite.

duty cycle. This requires a system that can automatically shut down when conditions are poor and restart when conditions improve. The thermal design minimizes power usage during the austral winter. This, in turn, reduces the number of batteries that must be transported to the field site (a major consideration). The overall strategy is to prepare an electronics enclosure that is heavily insulated and minimizes the volume that requires heating for the electronics such that the electronics themselves provide sufficient heat. The measurement sensors are rated to operate at the ambient exterior temperature.

A separate battery box is insulated with $10.1 \mathrm{~cm}$ of polystyrene panels and heated to between -15 and $-20^{\circ} \mathrm{C}$ during the austral summer for battery charging. The battery box is not heated during the winter to reduce power consumption. This is a chosen design consideration between degrading the capacity of the batteries at cold temperatures and using power to heat the batteries to achieve greater capacity. In this simple design, as the batteries cool while the system continues to draw several watts, the voltage will gradually drop. When the battery voltage drops below $11.2 \mathrm{~V}$, a low-voltage cutoff is activated and the system shuts down for the remainder of the winter. When, or if, this occurs depends upon the power used by the system (instruments, communications, and internal computer control) and the number of batteries. Increasing the number of batteries extends the operation of the system, but it requires additional transport. Since lead-acid batteries are very heavy, this is a significant trade-off consideration because of weight limits for the small aircraft used in the deployment. As it is, for the more distant deployment sites, several flights are required and fuel caches must be deployed to support the AAL-PIP installation.

The electronics box, illustrated in Fig. 4, is insulated using a combination of $10.1 \mathrm{~cm}$ vacuum panels and $10.1 \mathrm{~cm}$ of polystyrene panels. The actual box is shown in Fig. 5. The insulated volume inside the electronics box is heated yearround to $-27^{\circ} \mathrm{C}$. The measured thermal resistance of the electronics box is $0.07 \mathrm{~W}^{\circ} \mathrm{C}$, allowing the electronics box to maintain its interior temperature at $30^{\circ} \mathrm{C}$ above ambient by dissipating $2.1 \mathrm{~W}$ in the insulated volume.

The system utilizes six $40 \mathrm{~W}$ PV panels to charge the batteries during the period of daylight. When PV power is again available as the sun appears at the end of winter, the batteries are slowly heated to charging temperature. As the batteries warm up, their ability to deliver current increases and the remaining stored energy once again becomes available as the voltage rises. When the voltage rises to $11.8 \mathrm{~V}$ (typically around $-45^{\circ} \mathrm{C}$ ) the low-voltage detection circuit reconnects the load and the system resumes operation. In addition, a thermostat in the electronics box diverts power to a heater until the electronics reach operating temperature. In practice, 


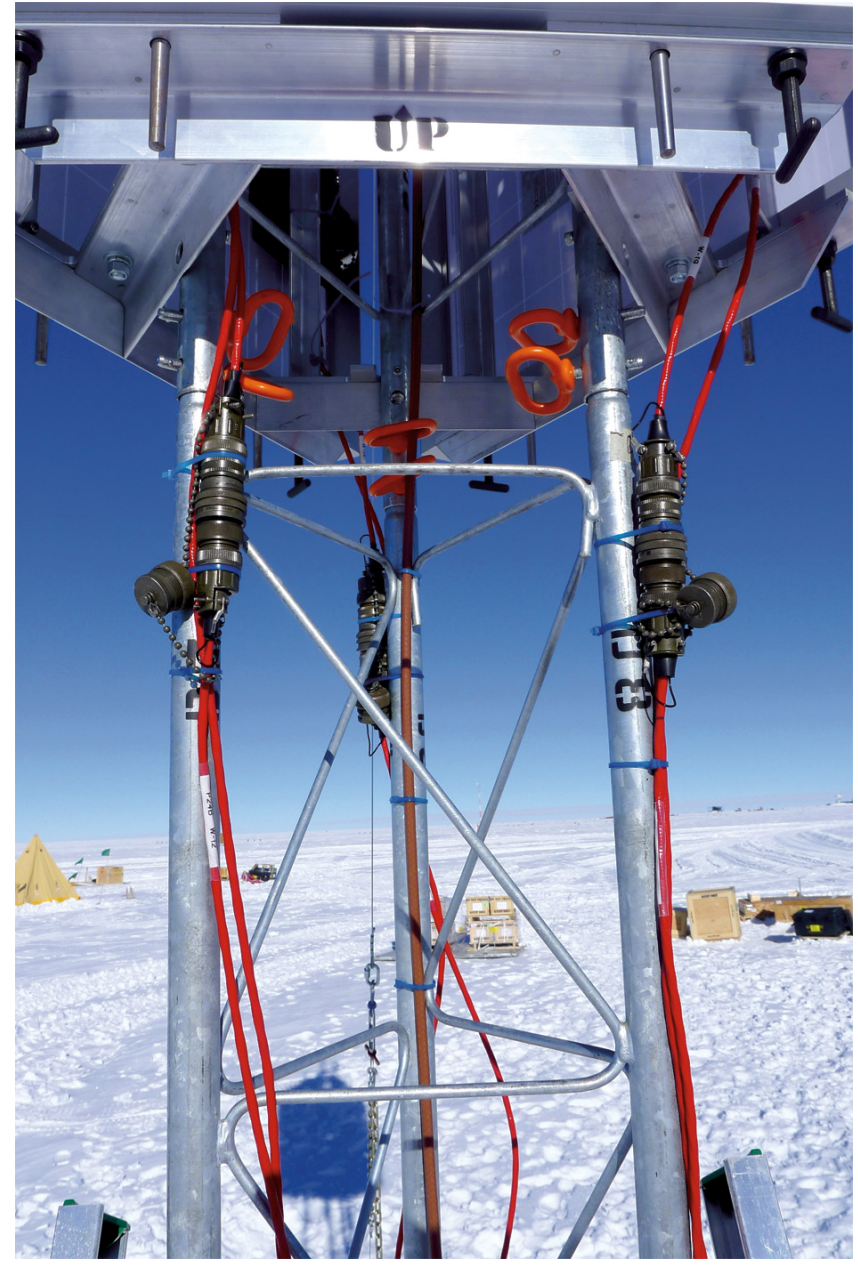

Figure 3. Tower close-up view showing orange pins used to secure tower sections and T-bolts at top securing the bottom of the PV panels.

the heater is powered only once during initial start-up. After that, the Iridium modem is power-cycled to control the electronics temperature.

Figure 6 shows, from the top, measurements of the temperature in the battery box; the temperature in the electronics box and the battery voltage for the AAL-PIP system, designated as Sys4 during testing at South Pole; and PG2 after deployment at a remote location on the East Antarctic Plateau. The data run from January 2011 through February 2014, and the system was deployed to the East Antarctic Plateau during the Antarctic summer field season: December 2012January 2013.

\subsection{Power subsystem}

In our earlier Greenland and Antarctic systems, we found that small wind turbines required yearly maintenance or replacement. This was mainly due to seizure of the bearings caused by grease loss and degradation. Since the AAL-PIP is

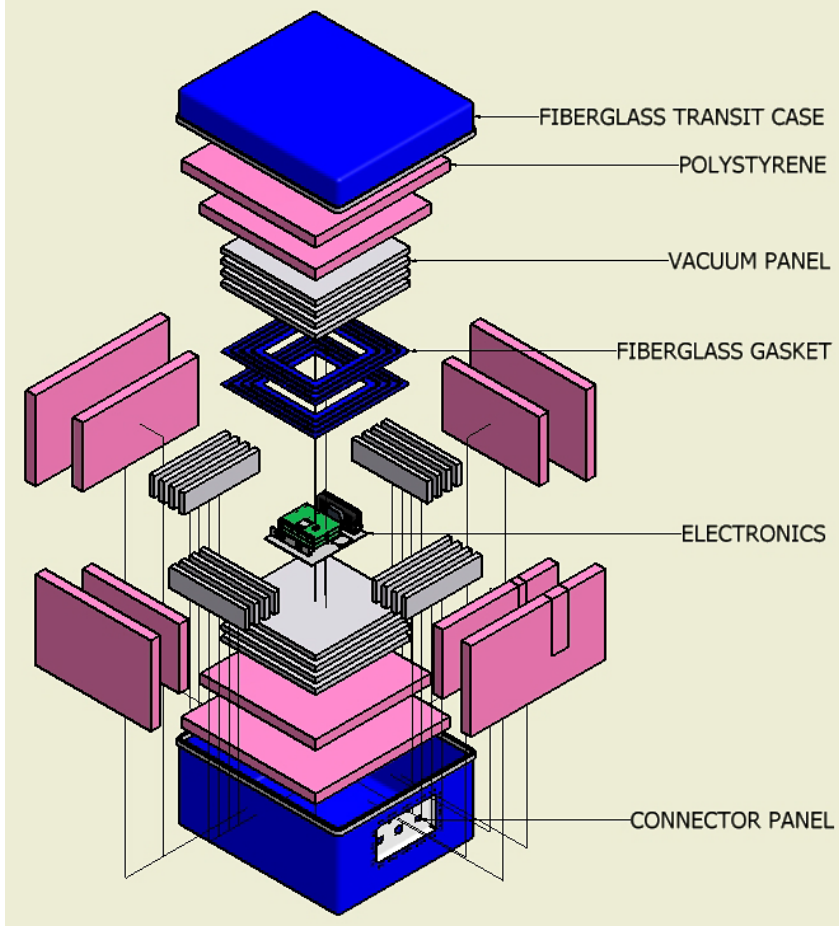

Figure 4. The super-insulated electronics box exploded view.

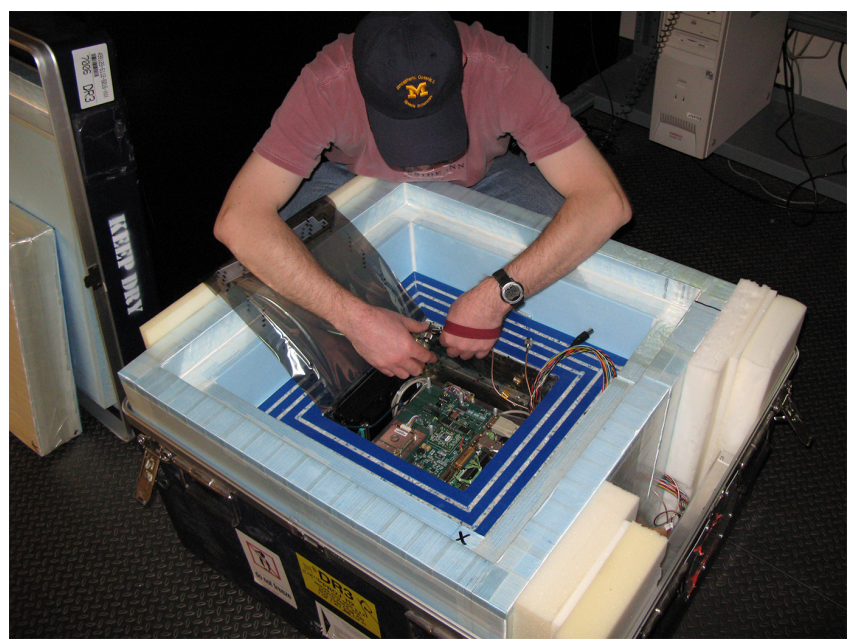

Figure 5. The super-insulated electronics box.

required to run for at least 5 years without maintenance, the use of wind turbines was ruled out, even though wind power is available year round in Antarctica while solar power is not. While the AGO platforms mentioned earlier have had success using wind generators, the AGO platforms also require more frequent maintenance visits, and we are not yet convinced that wind generators can be relied upon for long-term reliable operation in remote Antarctic conditions. 


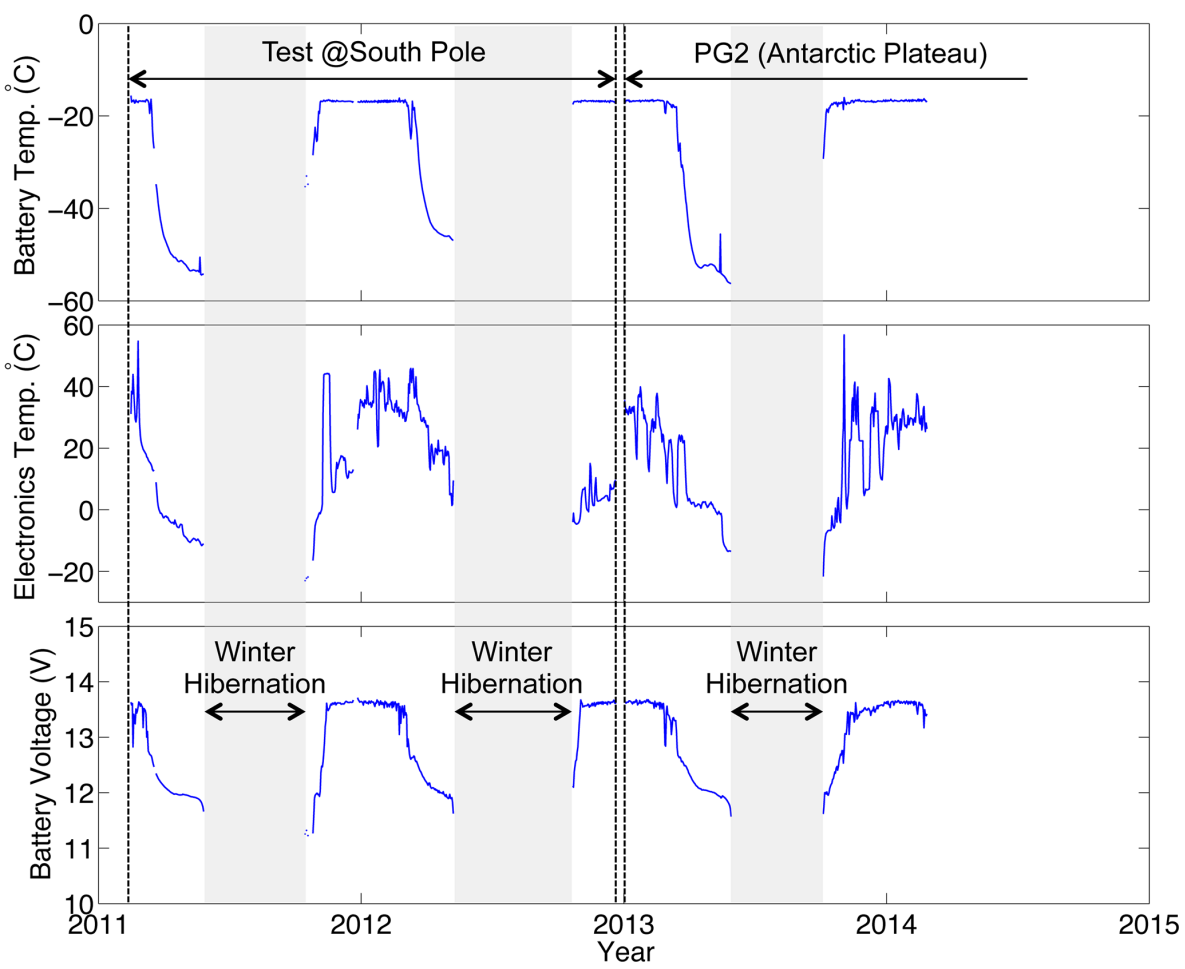

Figure 6. Measured temperature in the battery box (top), temperature in the electronics box (middle) and battery voltage (bottom) from 2011 to the end of February 2014. The AAL-PIP system was designated SYS4 during testing at South Pole station and renamed PG2 after deployment on the East Antarctic Plateau.

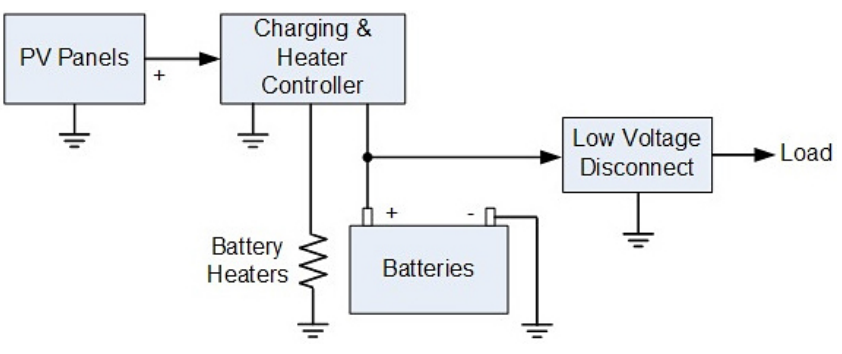

Figure 7. Power system block diagram.

Figure 7 shows a block diagram of the power system. The power system is designed around a bank of 16 100-amp-hour Power-Sonic absorbed glass mat (AGM) lead-acid batteries connected in parallel. Power-Sonic AGM batteries were selected because they have a proven track record of reliability and ruggedness in our earlier Greenland and Antarctica systems, and because they retain a significant amount of their rated capacity at low temperatures. In laboratory tests at the University of Michigan, a single Power-Sonic 100 amp-hour battery at $-55^{\circ} \mathrm{C}$ retained $48 \%$ of its rated capacity when powering a $1.4 \mathrm{~W}$ load. This allows us to power the system using unheated batteries during the austral winter, when the batteries cannot be charged due to lack of sunlight. The batteries are charged during the austral summer when PV power is plentiful. Since the battery temperature must be higher than $-20^{\circ} \mathrm{C}$ to accept a charge, the batteries are heated to between -15 and $-20^{\circ} \mathrm{C}$ while charging during the summer. Because the batteries operate at low temperatures, and because they undergo only one full charge/discharge cycle per year, we expect battery life to exceed 10 years. Our earlier design LPM system, which uses the same batteries and charge/discharge strategy, is in its 9th year of Antarctic operation with no signs of battery degradation.

Figure 8 shows the top layer of two layers installed in the battery box. Battery handling under Antarctic conditions (high altitude and cold) can be hazardous due to battery weight and possibility of electrical shorts. We minimize the danger by using the following components and procedures. The batteries are shipped with Delphi automotive connectors prewired to the battery terminals. The battery terminals are also coated with a silicone caulk before shipment to prevent electrical shorts. At the field site, the batteries are moved one at a time from the battery shipping containers to the battery box. They are then connected to the power control electronics using a single wiring harness. The wiring harness is built using Arctic Ultraflex wire (Power Wire Products, Inc.) and is fitted with mating Delphi connectors. The connection is made by snapping the two connectors together. No tools are required for the battery installation or connection, and it can be accomplished wearing gloves. Figure 9 shows the power 


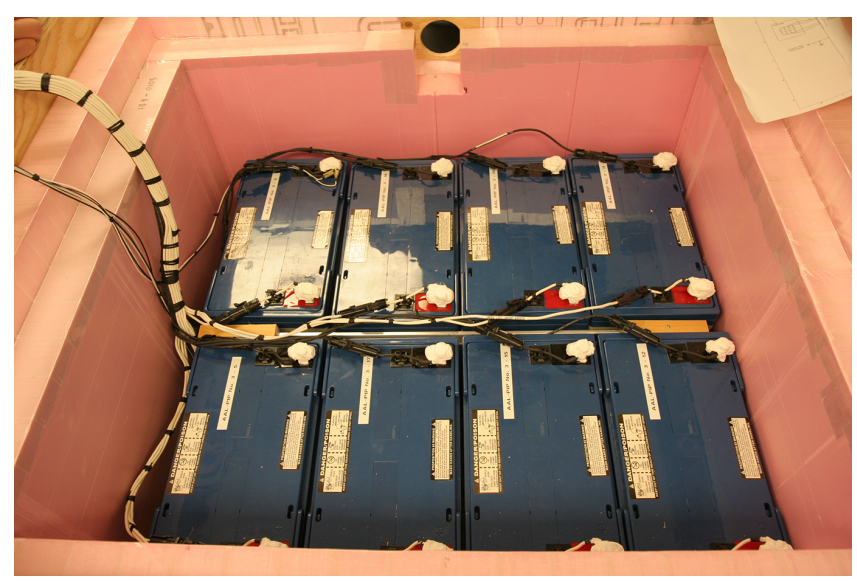

Figure 8. The top layer of two layers of batteries installed in the battery box. Note the aluminum battery heater plate between the two battery rows. Also note the custom wiring harness with Delphi connectors used to connect the batteries in parallel.

electronics box and fuse panel installed on top of the batteries in the battery box (left) and the power electronics printed circuit board inside the power electronics box (right).

Except for the PV panels and system power switch, the power system is contained entirely within the battery box, which is a heavy-duty plywood shipping crate lined with $10.1 \mathrm{~cm}$ of polystyrene insulation panels. To reduce the weight of the battery box for shipping and deployment, the batteries are shipped to the field site in separate foamlined plywood shipping crates, each containing two batteries. For shipment, the battery box contains PV panels and other lightweight and fragile items. The batteries are moved from their shipping crates to the battery box at the deployment site. In addition to the batteries, the battery box contains the power electronics board, fuse panel and battery heaters. The battery heaters are commercial power resistors mounted on an aluminum panel that is located in the center of the battery bank.

The power electronics board is a custom designed-printed circuit board that contains the battery heating, charging and low-voltage disconnect (LVD) circuits. A custom design was selected over an off-the-shelf solution for three reasons: low parasitic power losses, the need to control battery temperature and the extremely low operating temperature range. Since the AAL-PIP system depends on stored battery power for up to 6 months per year, parasitic power losses must be minimized. Off-the-shelf battery charge controllers have significant parasitic power losses and cannot control battery temperature. They also cannot operate down to $-70^{\circ} \mathrm{C}$.

The power electronics board performs the following functions:

1. sending all the PV power to the battery heaters when the batteries are too cold to charge
2. sending all the PV power to the batteries when the batteries are warm enough to charge and are not fully charged

3. disconnecting the PV panels when the batteries are warm and the batteries are fully charged

4. disconnecting the load (the electronics box) from the batteries when the battery voltage drops below $11.2 \mathrm{~V}$ and reconnecting the load when the battery voltage rises above $11.8 \mathrm{~V}$.

We note also that the PV panels never power the load directly. PV power is only used to heat and charge the batteries. The AAL-PIP system does not use mechanical relays or any other moving parts. Solid-state switches are used throughout. The heater control, charge control and LVD circuits all employ hysteresis to eliminate unnecessary switching cycles when voltages and temperatures approach threshold values.

\subsection{Computer control}

The control subsystem consists of a three-board PC-104 stack, an Iridium modem and antenna. It is contained, except for the Iridium antenna, in the electronics box. Figure 10 shows the PC-104 stack that comprises the core of the control system.

The PC-104 stack consists of a Technologics TS-7260 single-board computer (SBC), a Technologics TS-Ser4 quad serial port board and custom I/O board. The TS-7260 SBC has many features, including a Cirrus $200 \mathrm{Mhz}$ ARM9 32bit CPU, 64 MB RAM, 128 MB flash memory, Ethernet, two USB ports, three serial ports, digital I/O, 12-bit A/D converter, watchdog timer and battery-backed real-time clock, and an operating temperature range of -40 to $+85^{\circ} \mathrm{C}$. It runs the Linux operating system. A four-gigabyte flash drive connected to one of the USB ports provides mass storage for instrument and housekeeping data. A Garmin GPS receiver is used to provide an accurate time standard.

The only significant TS-7260 SBC deficiency for our application is inadequate system oscillator stability. This causes the system time to drift at a rate that varies strongly with the oscillator temperature. AAL-PIP is required to time-tag data with UTC $\pm 40 \mathrm{~ms}$, but uncorrected system time can drift several seconds per hour. System time stability could be improved by continuously powering the Garmin GPS receiver and synchronizing frequently, but in order to save power, we use a different method. The Garmin GPS receiver is powered up once per hour to synchronize system time to UTC. While synchronizing the system time, the system time error and drift rate are measured using the GPS pulse-per-second (PPS) signal. The system clock rate is then adjusted, using Linux kernel functions, to compensate for the error and drift rate. Using this method, the system time is consistently maintained at $\mathrm{UTC} \pm 5$ milliseconds. 

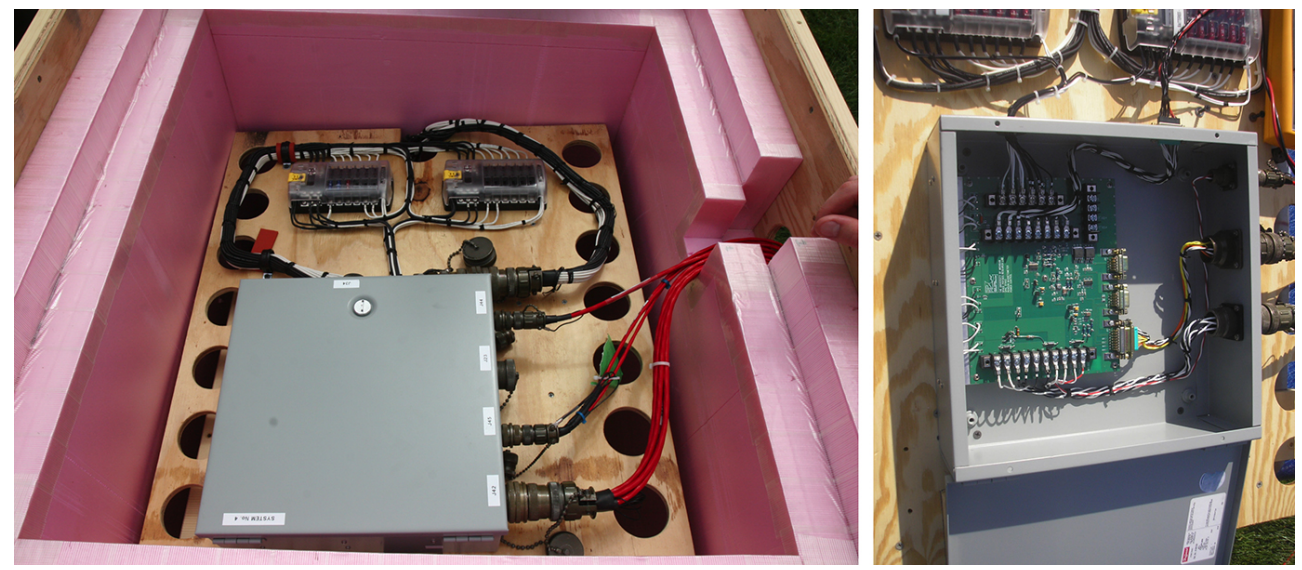

Figure 9. (left) Power electronics box and fuse panel installed on top of the batteries in the battery box. (right) Printed circuit board in the power electronics box.

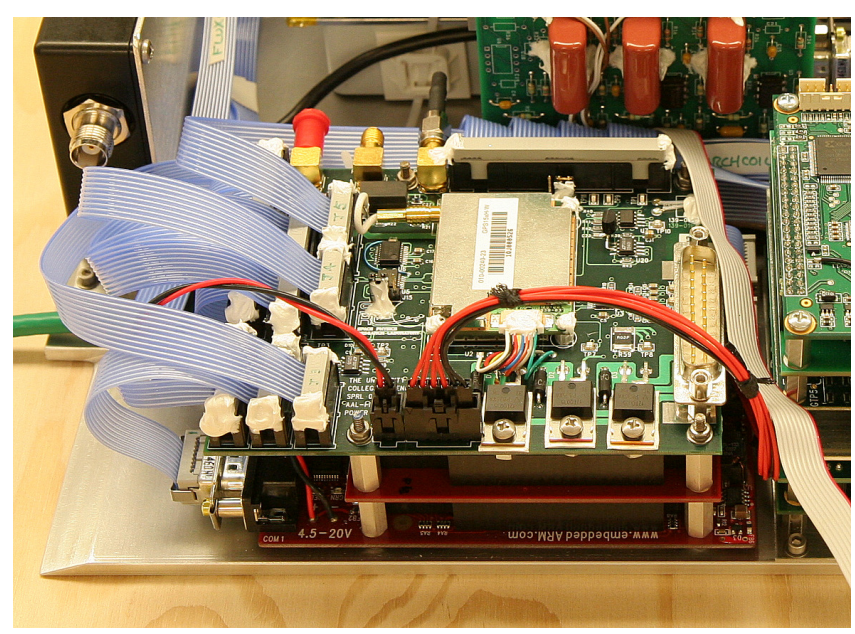

Figure 10. The PC-104 stack mounted on the electronics chassis. The custom I/O board is on the top.

The custom I/O board contains

- solid-state switches that control the Iridium modem, electronics box heater and instrument power.

- a Garmin GPS15H GPS receiver which transmits serial time messages to the TS-7260 SBC once per UTC second. It also transmits the digital PPS signal that is used to synchronize system time and measure time error and drift rate. The PPS signal is connected to one of the TS7260 SBC interrupt inputs.

- temperature, current and voltage sensors that are used to monitor battery voltages, battery temperatures, electronics temperature and electronics current.

- a warm-up thermostat which diverts power to a $5 \mathrm{~W}$ heater until the PC-104 stack reaches operating temperature. The stack is then powered up and a second software-controlled heater is used as needed to maintain the electronics temperature.

- GPS antenna power and signal splitting circuitry that powers the antenna and routes its RF output to the Garmin and the CASES GPS receivers.

- connectors and onboard interconnections that connect off-board instruments and peripherals to each other and the PC-104 stack.

The TS-Ser4 board contains four RS-232 serial ports which are used to communicate with the Garmin GPS receiver, Iridium modem, CASES GPS receiver and HF transceiver.

The TS-7260 SBC runs version 2.6.29 of the Linux operating system. The operating system distribution was created by Todd Valentic of SRI International and is customized for the TS-7260 SBC. The AAL-PIP control software is written primarily in the Python language, with hardware interface code written in $\mathrm{C}$. The software is structured as a set of independent processes. Each process is a separately running software program. Because Linux confines each process to its own memory space, if one process crashes, other processes are unaffected.

\subsection{Measured power usage}

The PC-104 stack, excluding the Garmin GPS receiver and antenna, requires $1.6 \mathrm{~W}$. The Garmin GPS receiver requires $0.4 \mathrm{~W}$. The Antcom dual-frequency GPS antenna requires $0.52 \mathrm{~W}$. The Iridium modem and antenna require a total of $0.9 \mathrm{~W}$ in standby mode. While transmitting, they require an average of 3 to $4 \mathrm{~W}$. The fluxgate magnetometer requires $0.8 \mathrm{~W}$. The search coil magnetometer requires $1.6 \mathrm{~W}$. The CASES GPS receiver requires $8.0 \mathrm{~W}$. The HF transceiver requires between 8 and $10 \mathrm{~W}$ while transmitting. 


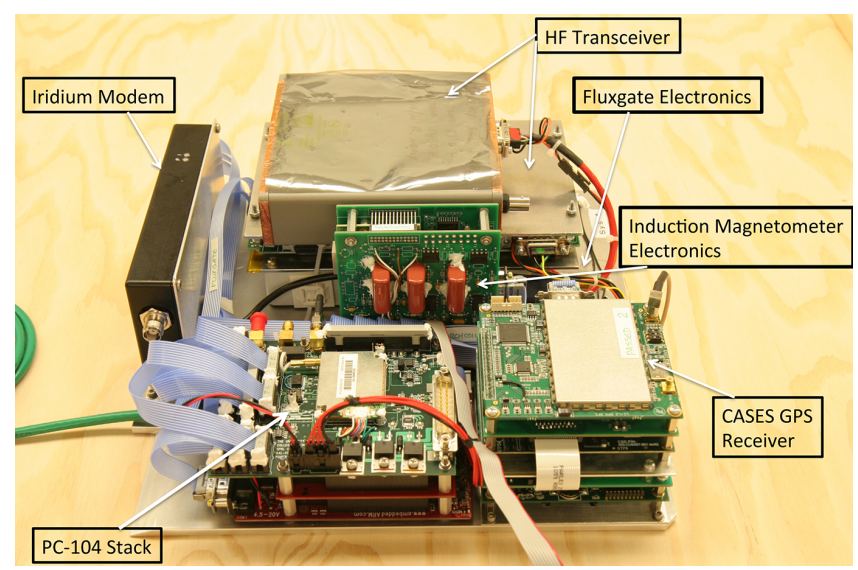

Figure 11. Electronics chassis.

\subsection{Sensor array subsystem}

The electronics for all of the sensors as well as the computer control and data handling are all enclosed in the superinsulated electronics box that is buried at the base of the tower during installation. Figure 11 shows the electronics chassis that is located in the electronics box.

\subsubsection{Fluxgate magnetometer}

The system supports a low-power, three-axis vector fluxgate magnetometer (LEMI-022AN) produced by the Laboratory for Electromagnetic Innovations (under the direction of Dr. Valery Korepanov), Lviv Centre of the Institute of Space Research, National Space Agency of Ukraine. It was specifically prepared to acquire stable three-component measurements of the Earth's magnetic field in the Antarctic region. The magnetometer consists of an electronic unit that is located in the super-insulated electronics box and a sensor with a connecting cable that is approximately $15 \mathrm{~m}$ in length. The main technical specifications of the instrument are given in Table A1.

\subsubsection{Induction magnetometer}

Induction magnetometers are widely used for space science research to measure magnetic field waves in space and on the ground. The magnetic field frequencies of interest in space physics typically fall into the ULF range (a few $\mathrm{mHz}$ to a few $\mathrm{Hz}$ ). Induction magnetic sensors are copper wires wound around a highly permeable $(\mu)$ metal core. A voltage is induced across the coil when magnetic field through the sensor changes over time $(\mathrm{d} B / \mathrm{d} t)$. Since induction magnetometers are sensitive to the magnetic field direction, it provides vector magnetic field information. The sensor is rated to operate at the ambient Antarctic temperature.

Each induction magnetometer deployed with the AAL-PIP system consists of two orthogonally mounted magnetic sen- sors with preamps, a cable of between 60 and $200 \mathrm{~m}$ and a main analog circuit. The two-axis magnetic sensor configuration provides measurements of wave activity in the geomagnetic north-south and east-west directions. The sensor is located between 60 and $200 \mathrm{~m}$ away from the AAL-PIP system to minimize interference from the electronics. Signals detected by the magnetic sensors are filtered and amplified by the main analog circuit, and archived in the AAL-PIP data acquisition system with 12 bit digitization at the rate of 10 samples $\mathrm{s}^{-1}$ axis $^{-1}$. The main analog electronics include amplifiers and band-pass filters, so that the magnetic field variation in the ULF range of interest can be detected. The magnetometer measures magnetic field wave activity with a resolution of tens of $\mathrm{pT} / \sqrt{\mathrm{Hz}}$ over the frequency response up to $2.5 \mathrm{~Hz}$ ( $-3 \mathrm{~dB}$ corner frequency). The power consumption of the induction magnetometer is $1.6 \mathrm{~W}$.

\subsubsection{GPS dual-frequency receiver}

The Connected Autonomous Space Environment Sensor (CASES) receiver is a scientific grade, low-cost, dualfrequency, GPS receiver developed by Cornell University, the University of Texas at Austin and Atmospheric \& Space Technology Research Associates (ASTRA). The development of CASES, its specifications and operation details are given by Crowley et al. (2011) and O'Hanlon et al. (2011). A space-qualified variant of CASES, called FOTON, has also been developed and is described by Lightsey et al. (2014).

This receiver is developed to be paperback-novel-sized, but its design was customized to a cubic form factor $(10 \mathrm{~cm} \times 10 \mathrm{~cm} \times 10 \mathrm{~cm})$ to fit inside the compact AALPIP electronics box. Furthermore, during the development, CASES passed the cold soak test below $-50^{\circ} \mathrm{C}$ and demonstrated reliable operation at about $-40^{\circ} \mathrm{C}$ before and after the test. The CASES receiver on an AAL-PIP system is connected to an Antcom GPS antenna. Power consumption is about $8 \mathrm{~W}$.

For CASES, all the data acquisition and tracking operations as well as science- and navigation-related operations, such as TEC computations, scintillation monitoring and calculation of navigation solution and GPS observables are performed on a general-purpose digital signal processor (DSP). Based on the real-time data processing by a scintillation monitor called "SCINTMON", for each tracking channel, the DSP outputs scintillation parameters $\mathrm{S} 4, \sigma_{\phi}$ and $\tau_{0}$ every 50-100 s; TEC, carrier phase and signal strength data at up to $10 \mathrm{~Hz}$; and high-rate, in-phase and quadrature correlation products with an unambiguous carrier phase reference at up to $100 \mathrm{~Hz}$ (Dierendonck et al., 1993; Humphreys et al., 2010, 2009). The data are then routed to a single-board computer that runs a server program for data logging and remote monitoring. As an ionospheric scintillation monitor, CASES has many advantages, including GPS L2 civil code (L2C) tracking capability, incorporation of specialized tracking loops designed for operation in both weak-signal and scintillating 
Table 1. Please provide a caption for Table 1.

\begin{tabular}{lr}
\hline Measurement range for each component & $\pm 65000 \mathrm{nT}$ \\
Cutoff frequency of analog low-pass filters (before digitization) & $0.3 \mathrm{~Hz}$ \\
Output data sample rate formed by averaging 8 AD conversions & $1 / \mathrm{s}$ \\
Resolution & $0.01 \mathrm{nT}$ \\
Temperature drift & $<0.5 \mathrm{nT}^{\circ} \mathrm{C}^{-1}$ \\
Spectral noise density at $1 \mathrm{~Hz}$ & $<10 \mathrm{pT}$ \\
Sensor component orthogonality error & $<30 \mathrm{~min}$ of arc \\
Temperature operating range for electronics & -40 to $+50^{\circ} \mathrm{C}$ \\
Temperature operating range for sensor & -65 to $+50^{\circ} \mathrm{C}$ \\
Power supply voltage & $12 \mathrm{~V} \pm 2 \mathrm{~V}$ \\
Power consumption & $<0.5 \mathrm{~W}$ \\
\hline
\end{tabular}

environments, and connectivity via a number of different options. CASES on AAL-PIPs may be accessed through an Iridium link for remote logging, reconfiguration and reprogramming.

As a part of real-time processing, SCINTMON implements a scintillation-triggering algorithm. A trigger indicates the presence of a phase or amplitude fluctuation more vigorous than the user-specified triggering threshold. In response to a triggering event, CASES collects high-rate data only for that duration, whereas low-rate data are saved at all times whenever CASES is powered up.

Because the maximum Iridium data download rate is approximately $16 \mathrm{MB}$ per day, data collected by all three space science instruments onboard - namely, the fluxgate magnetometer, the search-coil magnetometer and the CASES GPS receiver - need to be constrained. Each of the magnetometers collects about $1 \mathrm{MB}$ of data per day. Thus, in normal daily operation, CASES collects low-rate data and triggered highrate data until the daily memory limit for GPS data collection of about 12 to $13 \mathrm{MB}$ is reached. Its scintillation-triggering strategy enables CASES to make maximal use of its allotted daily data limit by capturing and passing along high-rate data only spanning the most vigorous scintillation events.

Furthermore, a special AAL-PIP "storm mode" can be enabled in anticipation of an incoming solar storm. In storm mode, CASES is allowed to store much more than the standard daily data limit. Data stored locally in storm mode are retrieved over a period of several days post-storm.

Finally, CASES employs a temperature-compensated crystal oscillator (TCXO) as its reference oscillator. Although this feature causes CASES to be less expensive than most of the commercially available scintillation monitors, its usage introduces receiver clock errors which themselves can cause false detection of phase scintillation. However, receiver clock errors are removed in real time within SCINTMON by differential processing before SCINTMON applies its scintillation-triggering tests, and they can be removed by post processing the data as described by Deshpande et al. (2012).

Geosci. Instrum. Method. Data Syst., 3, 211-227, 2014

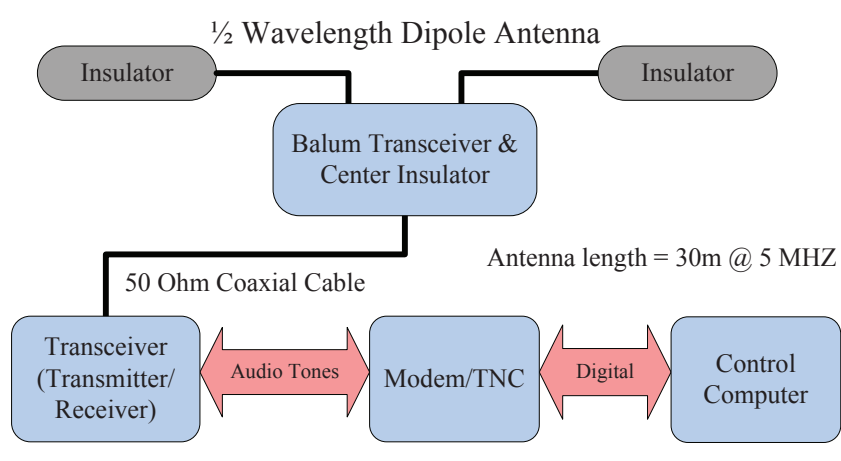

Figure 12. HF radio system.

\subsubsection{HF radio experiment}

At distances beyond a few kilometers HF radio propagation is an ionospheric phenomenon (Hunsucker and Bates, 1969; Blagoveshchensky et al., 2008). In the lower HF band a critical frequency can exist where a wave launched vertically is reflected. Frequencies near that critical frequency are useful for communication at distances up to several hundred kilometers. Under normal propagation conditions signal levels are typically strong and consistent. Propagation is enhanced by strong $\mathrm{F}$ region ionization. Strong ionization in the D region, however, attenuates the signal so that the optimum signal frequency varies toward higher frequencies during daylight hours but is reduced during night. This effect is further enhanced during geomagnetic storms when particle precipitation and X-ray flux levels increase (Warrington et al., 2012). An interesting aspect of this propagation mode is that it is enhanced when the antenna is designed to direct the maximum energy at high (nearly vertical) angles rather than toward the horizon. This mode is sometimes referred to as near vertical incidence sky wave (NVIS) propagation. High-latitude statistics on HF propagation over distances of hundreds of kilometers, particularly in the polar Antarctic region, are not well developed, so we include a HF radio experiment to collect information about propagation statistics between our array of stations (Collin, 1985). 
The HF radio system (shown in Fig. 12) consists of three major modules: antenna, radio transceiver and modem. The antenna is a half-wave horizontal dipole antenna designed to operate at $5 \mathrm{MHz}$ frequency. The antenna is constructed of wire supported approximately $1.5 \mathrm{~m}$ above the ice. Since the ice is relatively transparent to electromagnetic radiation at this frequency, the antenna height is not critical. Additionally, as the propagation is by way of sky wave (reflected from the ionosphere), the horizontal dipole best facilitates the high angle radiation direction required for relatively short distance HF propagation. The transceiver is a fixed frequency SSB radio which converts the radio frequency to and from the audio tones of the modem. The radio transmissions are very low power at approximately $3 \mathrm{~W}$. The modem is a DSP-based device capable of numerous modes of operation. The modem interfaces with the AAL-PIP system control computer which controls radio operation and handles data through a serial data link.

The normal modulation mode is frequency-shift keying (FSK) at $100 \mathrm{Bd}$. The protocol is Simplex Teletype Over (RadioSITOR-B), which is a maritime HF communication standard using forward error correction (FEC). The relatively low data rate is designed to provide maximum effectiveness in the presence of HF noise and fading. Narrow-bandwidth filters (approximately $300 \mathrm{~Hz}$ ) in the modem and transceiver provide a high signal-to-noise ratio which, along with the FEC, offsets much of the disadvantage of the low-power transmitter.

During operation of the HF antenna, the AAL-PIP computer applies a lookup table according to the system temperature to maintain the transceiver frequency within $50 \mathrm{~Hz}$ of the correct channel frequency. The frequency correction process is implemented by shifting the frequency of the modem audio tones since this may be done in small increments. The transceiver and modem have been tested for operation over a temperature range of -40 to $50^{\circ} \mathrm{C}$ with a cold soak below $-50{ }^{\circ} \mathrm{C}$

While SITOR-B provides excellent communications in the radio system, FEC serves to mask some errors in the communication link. The modem provides several additional communication modes, some of which do not provide any error correction. The use of non-error-corrected modes can allow for experiments which better indicate the channel characteristics. Selection of mode, protocol and data rate and transmitted power level are under software control, so many experiments are possible.

The locations of the AAL-PIP systems in Antarctica as of January 2014 is shown in Fig. 13. In current experiments, the HF radio system is briefly turned on at $2 \mathrm{~h}$ intervals for test transmissions. Each station transmits in turn, with the other stations receiving. The transmission consists of several repetitions of a telegraphic test symbol (vvv) and the station identifier in plain text. Since the exact transmitted pattern is known, a character error rate can be found for each transmission. Initial results for tests between a site on the Antarctic plateau and the South Pole station (a distance of approximately $600 \mathrm{~km}$ ) indicate very high communication reliability except during periods of high $\mathrm{D}$ region absorption due to proton events. During significant proton events the signal reliability drops rapidly to zero as the $5 \mathrm{MHz}$ signals are highly attenuated while passing through the $\mathrm{D}$ region. This suggests that the absence of signals is a good indicator of a significant event.

An example of D-region attenuation during geomagnetic storm periods in the 2011-2012 austral summer is shown in Fig. 14. Two major storms were evident during this season. The first occurred towards the end of January 2012, and the second occurred during the first part of March 2012. In both storm cases, the HF communication links between the installed systems were severely affected, with periods of total communication "blackout" witnessed during the height of the storms. There are also periods of communication disruption evident during minor storm events occurring in the same season. With the completion of the 2013-2014 summer season, many of the systems have been aligned along the geomagnetic field line, creating a much improved geometry for the study of HF communication impacts during geomagnetic storm time conditions in Antarctica.

\section{Data handling}

Because the AAL-PIP systems are extremely remote, maintaining Iridium communications is the highest software priority. Loss of communications means an expensive and timeconsuming field trip. The communications software processes are well-tested, mature and independent of the other processes. Any of three different watchdog timers can force a hard reset if the Linux kernel crashes or communications are lost for a predetermined length of time. This scheme has proven reliable. We have never lost communication with an AAL-PIP system due to a software bug.

In previous systems, we used Iridium modem-to-modem connections for communications. We now use an Iridium protocol known as RUDICS (Router-based Unrestricted Digital Interworking Connectivity Solutions), which is a combination of a single Iridium modem and the Internet to provide communication between a remote system and an Internetconnected computer. RUDICS has several advantages over the modem-to-modem communications:

- fewer line drops because only one modem is used

- a single Internet-connected computer can handle many RUDICS connections simultaneously

- lower cost because only one modem per remote system is required.

Each remote AAL-PIP system initiates a RUDICS connection with the operations center computer located at the 


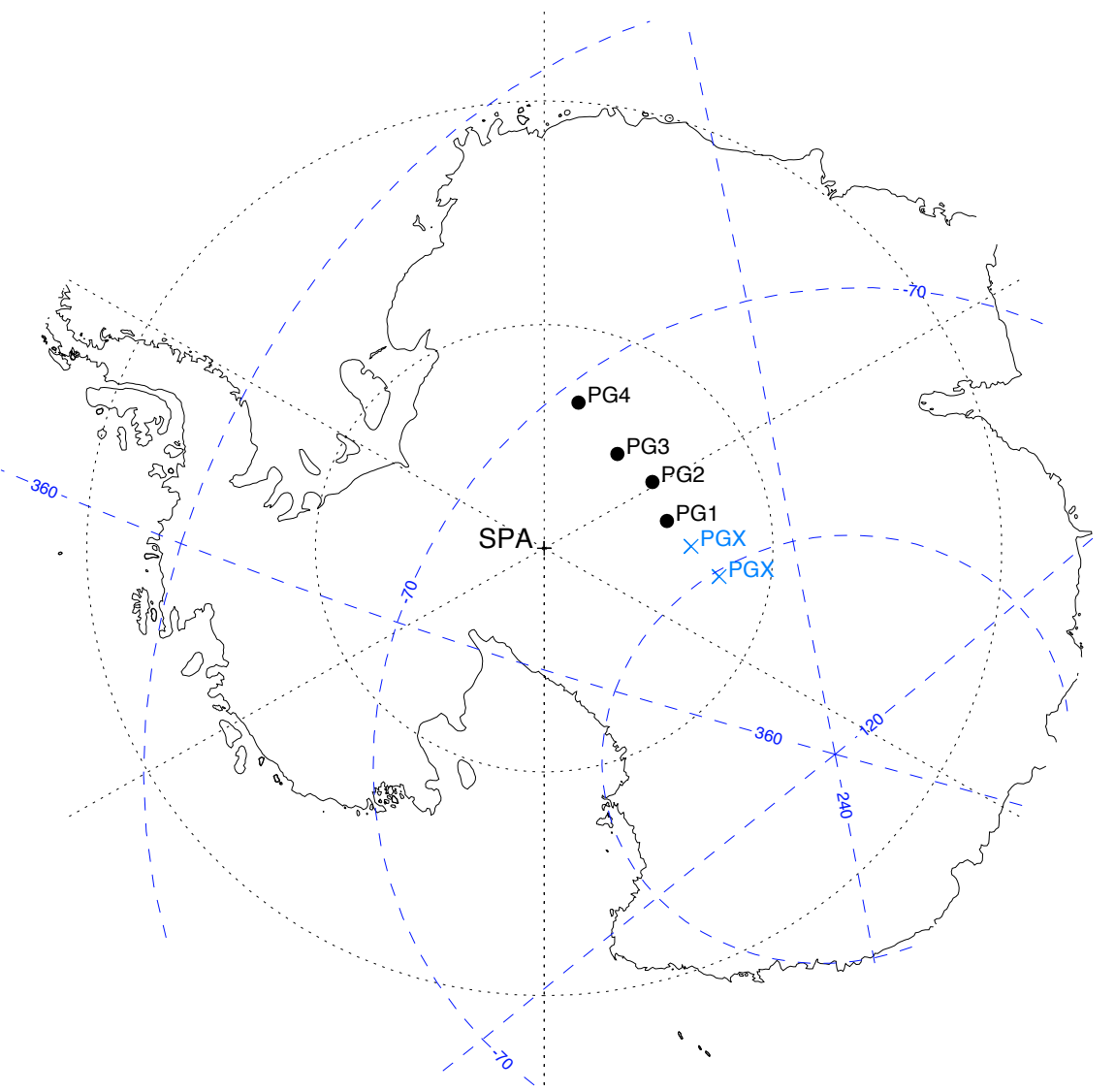

Figure 13. Map showing the locations of the AAL-PIP systems deployed in Antarctica since January 2014.

University of Michigan (UM) every $30 \mathrm{~min}$. If the connection is idle for more than $5 \mathrm{~min}$, the AAL-PIP terminates the connection to conserve power. Continuous contact with an AAL-PIP system can be maintained by executing a script on the operations center computer that pings the AAL-PIP system periodically. When the AAL-PIP system initiates a connection to the DOD Iridium gateway in Hawaii, the gateway then connects the AAL-PIP system to the operations center server using Internet and TCP/IP (Transmission Control Protocol/Internet Protocol) socket protocol. It typically takes about $40 \mathrm{~s}$ for an AAL-PIP system to connect to the server. One server can handle many RUDICS connections simultaneously. The RUDICS link provides about 2000 bits s $^{-1}$ of half-duplex raw data throughput.

From the operations center server we can

- open one or more Unix Secure Shell (SSH) terminal sessions on the AAL-PIP

- automatically download data files for any set of instruments and dates.

We use SSH sessions to acquire diagnostic information and update AAL-PIP software. Updating the AAL-PIP software is done by copying two files to a particular AAL-PIP directory and rebooting the TS-7260 SBC. The remote SSH session and software-updating capabilities have proven invaluable.

The operations center server automatically runs a data download program at 00:15 UTC daily. Typically, all of the data stored the day before are downloaded and then erased from the AAL-PIP flash drive. The download program can also be configured to download data for any subset of instruments and range of dates.

Iridium communications are subject to intermittent data corruption and dropped connections, so an error detection and retransmission protocol is required for automated file downloading. We are using a custom protocol to maximize performance over the relatively slow, unreliable Iridium link. Large blocks of data are transmitted to minimize protocol overhead and communications line turnarounds. Using this custom protocol, file download throughput approaches the link speed of 2000 bits s $^{-1}$.

Following the acquisition of the raw telemetry data by the operations center computer at UM, the data files are transferred to the Virginia Tech Web server that is hosted on a desktop computer. Here the files are converted into formats that are more readily accessible for scientific analysis, plotted, archived and made available on the Web and for file transfers. These tasks are accomplished by a combination of 

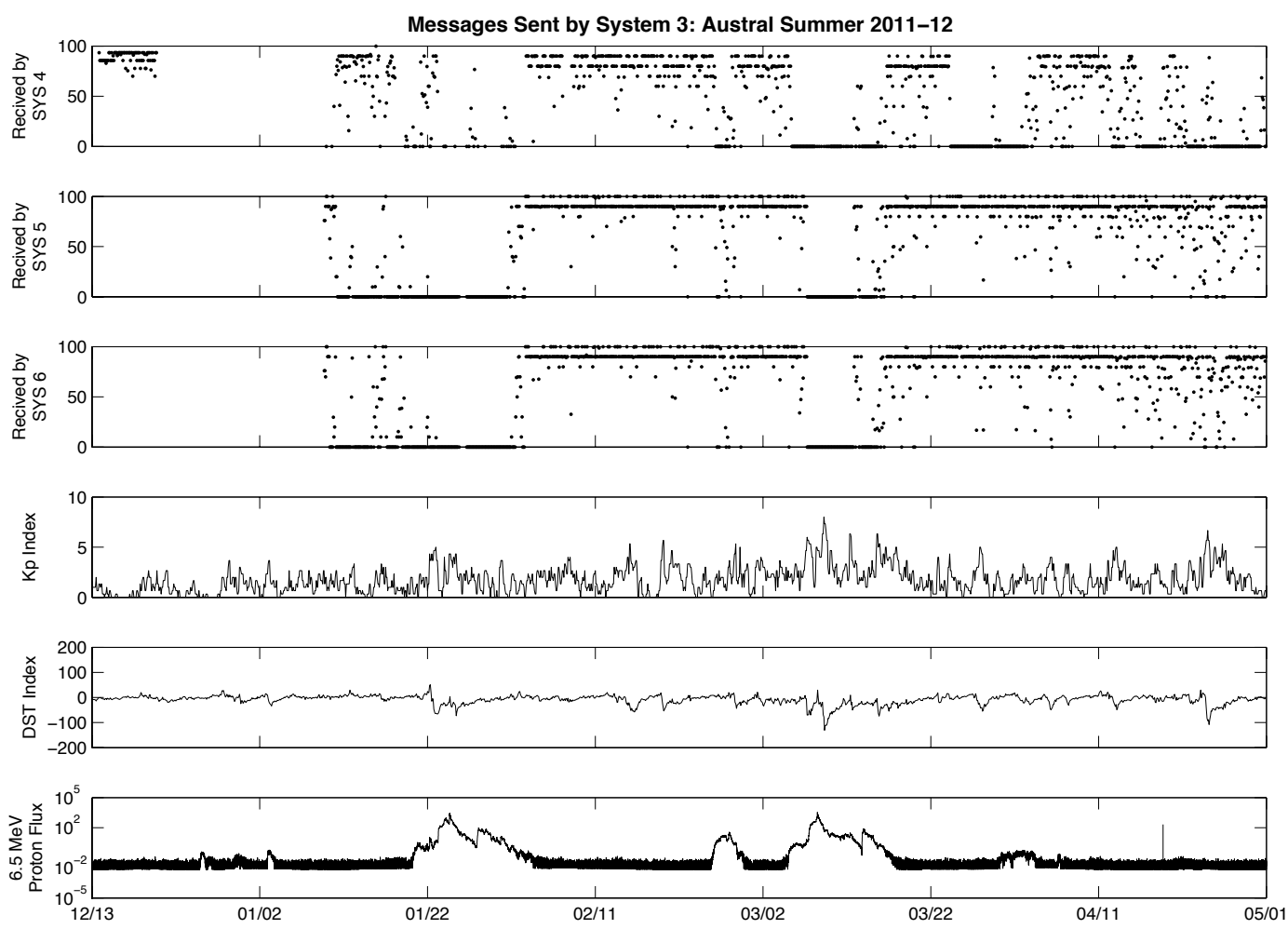

Figure 14. HF communication quality during the 2011-2012 summertime period. The top three panels indicate the percentage of characters received correctly as transmitted from system 3 to systems 4-6 (see Fig. 13 for a map of the location of the varying systems during this time frame) versus time. A level of 100 indicates that all receptions were error free. A level of 0 indicates that all of the receptions included a complete distortion of the transmitted file character set. The bottom three panels plot the $K_{\mathrm{p}}$ index, $D_{\mathrm{ST}}$ and proton flux $\left(\# /\left(\mathrm{s}-\mathrm{cm}^{2}-\mathrm{sr}\right)\right)$ over the same time period. All systems assumed nominal operations from 10 January 2012 until the end of the season (May 2012).

Unix Bash shell scripts, Python programs and IDL (Interactive Data Language) programs.

A shell script that starts the processing is executed automatically at fixed intervals, twice per day. A Python program connects to the SFTP server at UM, checks the data directory contents and downloads any new data files that are not already on the local file system. Sometimes there are no new files, such as when the data transfers are suspended during the Antarctic winters and no solar power is available. If new files are found then, following their download by SFTP (Secure File Transfer Protocol), the list is handed off for further processing by IDL programs.

The first step in the processing is conversion of the files into a "flat file" format that in principle can be accessed on any computing platform in any language. As the raw AALPIP data do not contain fixed time intervals, there are multiple files from each instrument for each day (approximately 24 per day), and these files need to be sorted and concatenated in the proper sequence to obtain a new, 1-day file.

Traditionally, flat files up through version 3 have used two separate files to hold an ASCII (American Standard Code for Information Interchange) text "header" and binary data. A newer, version 4 format is now used in which the text header and binary data are combined into one file. The header contains a description of the data contents, in a standardized format that both is human readable and can be used by programs for further processing. The binary data begin at a fixed offset from the start of the file; this offset value is included in the text header but is normally 4096 bytes, which allows adequate space for most headers. The byte order of the data may be either big or little endian and is specified in the header. Programs that read flat files should be able to handle both formats. The flat files are stored in a database having subdirectories for each year and month, but with all magnetometer sites combined together.

Following the conversion to flat files, the new data are graphed, with the results saved in both PDF and PNG formats. The data are also converted to the Common Data Format (CDF). A Web page (http://mist.nianet.org) shows the most recent plots, and also has links to the archived plots and access to the CDF data files via HTTP. FTP access to the flat files is also available to the research group and collaborators. Due to the risk of hacker attacks that could result from "anonymous" FTP access, our FTP server requires a user name and password. These are made available to collaborators. Copies of the CDF data files are also available 


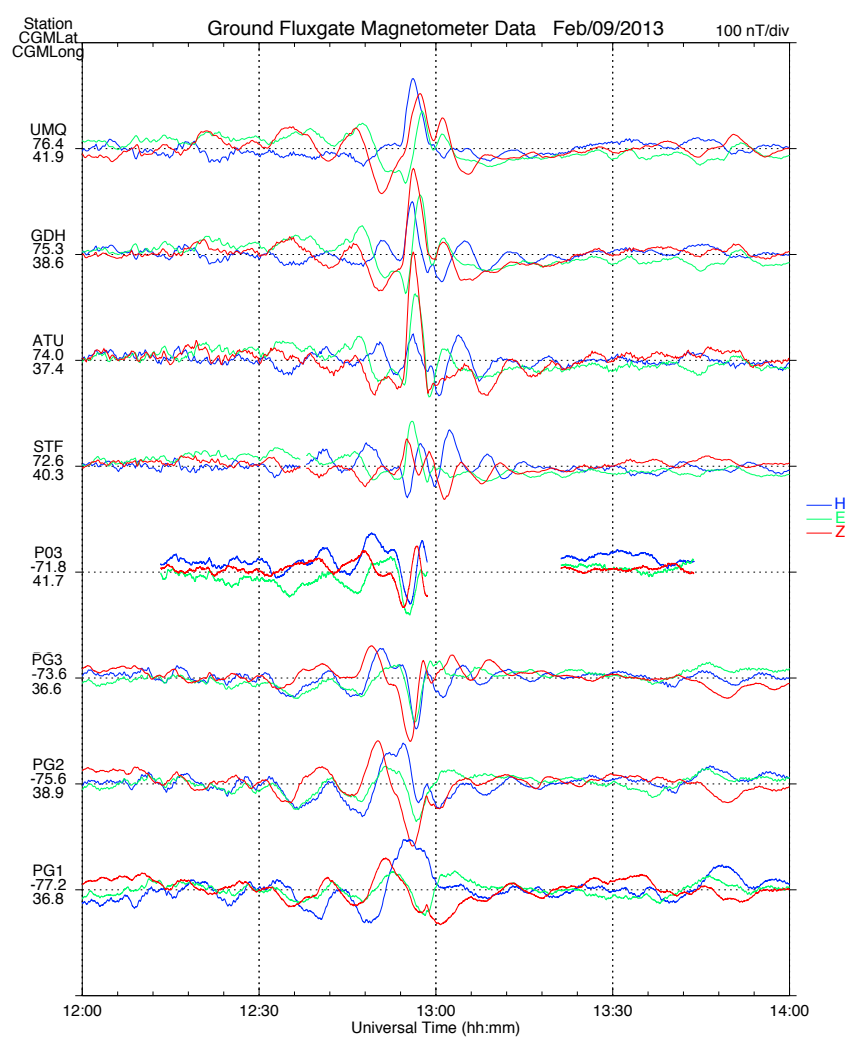

Figure 15. Geomagnetic impulse events observed by the fluxgate magnetometers at four station pairs in magnetically conjugate hemispheres (UMQ-PG1, GDH-PG2, ATU-PG3 and STF-P03) starting approximately at 12:45 UT on 9 February 2013.

in the THEMIS (Time History of Events and Macroscale Interactions during Substorms) database (Angelopoulos, 2008; Mende et al., 2008). The THEMIS project obtains these files automatically through FTP.

Backups of the data files and processing programs are maintained on a redundant, RAID system (redundant array of independent disks; the present capacity is 2 TB). All programs are also backed up on another system, normally through the use of DropBox. As the computer system that does the final processing and archiving of the data is accessible over the Internet for HTTP and FTP transfers, there is some exposure risk to malicious attacks (the firewall log shows multiple attempts daily). For this reason the RAID backup file system is not continuously connected to the host computer, ensuring that it would not be affected by a successful attack; backups to the RAID file system are done manually.

Figures 15 and 16 show example events from the data obtained by the AAL-PIP systems located in East Antarctica (PG1, PG2, and PG3). See the map in Fig. 13 for the locations of the remote field stations. Geomagnetic disturbances are clearly seen in Fig. 15, in which four station pairs in magnetically conjugate hemispheres (UMQ-PG1, GDH-

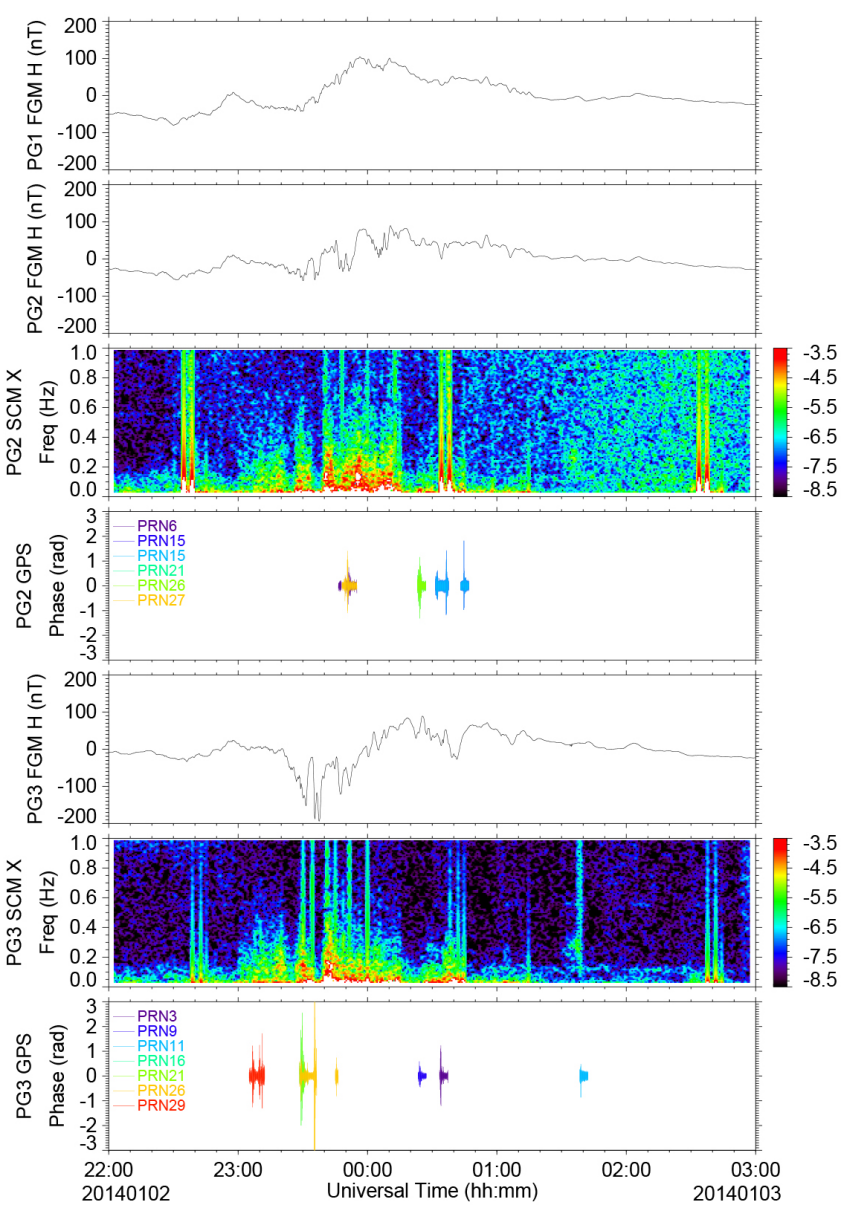

Figure 16. Measurements at the three remote field stations (PG1, PG2 and PG3) from 22:00 UT on 2 January 2014 to 03:00 UT on 3 January 2014 showing magnetic field disturbances and associated with GPS signal sintillations observed by (a) PG1 fluxgate magnetometer; (b-d) PG2 fluxgate and induction magnetometers, and GPS scintillation monitor; and (e-g) PG3 fluxgate and induction magnetometers, and GPS scintillations monitor. Note that the magnetic field data are measured from the $\mathrm{H}$ (fluxgate magnetometer) and $\mathrm{X}$ (induction magnetometer) components, both of which are aligned along the horizontal local magnetic field line.

PG2, ATU-PG3 and STF-P03) observed sudden changes in magnetic signatures (called "magnetic impulse event") starting approximately at 12:45 UT. It is found, however, that there is asymmetry in the patterns as well as the amplitudes of the magnetic events. Kim et al. (2013) suggest that asymmetry in ground response patterns between the conjugate locations often shows little correlation with interplanetary magnetic field (IMF) orientation, season and ionospheric conductivity, indicating that a much more complex mechanism might be involved in creating interhemispheric conjugate behavior.

As shown in Fig. 16, geomagnetic field disturbances called "substorm" in association with ionospheric scintillations are observed by (a) the fluxgate magnetometer at PG1; (b-d) 
fluxgate and induction magnetometers, and GPS scintillation monitor at PG2; and (e-g) fluxgate and induction magnetometers, and GPS scintillation monitor at PG3. The substorm is a disturbance in the Earth's magnetosphere causing energy to be transferred from the tail side of the magnetosphere to the high-latitude ionosphere. This is accomplished through the precipitation of energetic particles along magnetic field lines into the atmosphere producing the aurora, and through an intensification of electrical current flowing in the highly conducting ionospheric auroral electrojet. GPS scintillations are often observed during geomagnetically disturbed times (Prikryl et al., 2011; Kinrade et al., 2012). Investigating interhemispheric comparisons of bipolar GPS scintillation maps, Prikryl et al. (2013) have reported that the scintillation occurrence is significantly higher in the southern cusp and polar cap compared to the northern regions. However, they also mention that the coverage of GPS receivers was insufficient to study the relation of IMF to the strong hemispherical assymmetry in the intensity of scintillations.

As shown here in the fluxgate magnetometer data in Fig. 16, the onset of the substorm event occurred at 23:20 UT. The spectrograms from the induction magnetometers (panels $\mathrm{c}$ and $\mathrm{f}$ ), showing wave power as a function of frequency over time, display broadband pulsations during the substorm event. This is a very well known manifestation of substorms. The event also appears to be associated with the phase scintillations in the GPS signals (panels $\mathrm{d}$ and g). It should be noted that the GPS receivers were turned on from 22:00 to 03:00 UT. Similar results have been reported by Kim et al. (2014), showing a possible connection of ionospheric irregularities to a substorm event using simultaneous observations of ULF waves and GPS scintillations.

\section{Conclusions}

We have learned a number of lessons over the years through our engagement in remote polar measurements. With regard to the AAL-PIP system described here, the glove-friendly, tool-free deployment design has proven to be very well thought out and highly valued by the members of the deployment teams. This is one of the most innovative and valuable features of this new design.
The support of multiple instruments has increased the demand for power, with the consequence that the system spends a longer time in winter hibernation mode than with previous designs. New strategies for instrument operation or supplying additional batteries could help to reduce the hibernation period. The system has been designed such that the battery box is a modular unit and it is simple to add more battery boxes to the system. The limitation, however, is the ability for logistical support to accommodate the weight and transport of the batteries to the remote sites.

The system, as it is deployed now, provides no outputs for the deployment team to monitor, other than to see that the power is on. The orientation of the magnetic sensor is done by using an Iridium phone to talk with a person in the laboratory at Virginia Tech who is monitoring the data transmitted by the station. The magnetic sensor is rotated to place the $x$ axis along the magnetic field by nulling the $y$ axis ( $X$ and $Y$ lie in the horizontal plane, and $Z$ is positive vertical in the down directions). This has been satisfactory for the deployment of the magnetometer and gives a good end-to-end test of the system. However, if odd readings are obtained from any of the instruments or other components of the system do not operate as expected, it is difficult for the deployment team to undertake any simple diagnostic steps - checking cables, connectors, voltages, etc. It would be useful to have some type of diagnostic system output available to the deployment team for such purposes.

Our concern for operating in the cold environment using the super-insulated electronics box provided a surprise when the temperature of the electronics got above $40^{\circ} \mathrm{C}$ during summertime with extended operation of the CASES GPS receiver. The result has been that we have needed to limit the operation of the receiver because of temperature considerations within the electronics box. We are able to heat the volume containing the electronics, but we have no way of actively cooling the volume. More thought must be given to this problem. 


\section{Appendix A}

Table A1. Web resources for autonomous polar system design.

Wisconsin Automatic Weather Station Project
McMurdo Long Term Ecological Research (LTER)
IRIS/PASSCAL Polar Seismology
Greenland Ice Sheet Monitoring Network
UNAVCO Polar Geodetic Support
Power Systems for Polar Environments
Polar Technology Conference
POLENET project site
Antarctic PENQUIn Program
Augsburg College Space Physics
British Antarctic Survey Instrumentation
Virginia Tech Magnetosphere - Ionosphere Science Team
Autonomous Polar Observing Systems Workshop Report

http://amrc.ssec.wisc.edu/aws/

http://www.mcmlter.org/

http://www.passcal.nmt.edu/content/polar/

http://glisn.info/

http://www.unavco.org/polartechnology

http://www.polarpower.org

http://www.polartechnologyconference.org/

http://www.polnet.org/

http://www.sos.siena.edu/antarctic/PENGUIn_Program

http://space.augsburg.edu/index.html

http://www.antarctica.ac.uk/bas_research/instruments/index.php

http://mist.nianet.org/

http://www.iris.edu/hq/files/publications/other_workshops/docs/APOS_FINAL.pdf 
Acknowledgements. Support for the development and testing of this system has been provided through Major Research Infrastructure (MRI) Grant ATM-922979 to Virginia Tech from the National Science Foundation. Additional support has been provided by the National Science Foundation for the operation and scientific investigation of data from the deployed AAL-PIP stations along the Antarctic $40^{\circ}$ magnetic meridian by grants ANT-08398585 and PLR-1243398. Support at the University of Michigan has been provided by NSF grant ANT-0838861 and support at ASTRA has been provided by NSF grant PLR-1243225.

Edited by: L. Eppelbaum

\section{References}

Angelopoulos, V.: The THEMIS mission, Space Sci. Rev., 141, 534, 2008.

Blagoveshchensky, D. V., Kalishin, A. S., and Sergeyeva, M. A.: Space weather effects on radio propagation: study of the CEDAR, GEM and ISTP storm events, Ann. Geophys., 26, 1479-1490, doi:10.5194/angeo-26-1479-2008, 2008.

Collin, R. E.: Antennas and Radiowave Propagation, McGraw-Hill, New York, 1985.

Crowley, G., Bust, G. S., Reynolds, A., Azeem, I., Wilder, R., O’Hanlon, B. W., Psiaki, M. L., Powell, S., Humphreys, T. E., and Bhatti, J. A.: CASES: A novel low-cost ground-based dualfrequency GPS software receiver and space weather monitor, in: Proc 24th International Technical Meeting, Satellite Div. of the Institute of Navigation (ION GNSS 2011), 1437-1446, Inst. of Navigation, Manassas, VA, 2011.

Deshpande, K. B., Bust, G. S., Clauer, C. R., Kim, H., Macon, J. E., Humphreys, T. E., Bhatti, J. A., Musko, S. B., Crowley, G., and Weatherwax, A. T.: Initial GPS scintillation results from CASES receiver at South Pole, Antarctica, Radio Sci., 47, RS5009, doi:10.1029/2012RS005061, 2012.

Dierendonck, A. J. V., Klobuchar, J. A., and Huai, Q.: Ionospheric scintillation monitoring using commercial single frequency C/A code receivers, in: Proc of ION GPS-93, p. 1333, Inst. of Navigation, Manassas, VA, 1993.

Humphreys, T. E., Psiaki, M. L., Hinks, J. C., O’Hanlon, B., and Kintner, P. M.: Simulating ionosphere-induced scintillation for testing GPS receiver phase tracking loops, Selected Topics in Signal Processing, IEEE J., 3, 707-715, 2009.

Humphreys, T. E., Psiaki, M. L., and Kintner, P. M.: Modeling the effects of ionospheric scintillation on GPS carrier phase tracking, Aerospace and Electronic Systems, IEEE Trans., 46, 1624-1637, 2010.

Hunsucker, R. D. and Bates, H. F.: Survey of polar and auroral region effects on HF propagation, Radio Sci., 4, 347-365, doi:10.1029/RS004i004p00347, 1969.

Kadokura, A., Yamagishi, H., Sato, N., Nakano, K., and Rose, M.: Unmanned magnetometer network observation in the 44th Japanese Antarctic Research Expedition: Initial results and an event study on auroral substorm evolution, Polar Science, 2, 223 235, doi:10.1016/j.polar.2008.04.002, 2008.

Kim, H., Cai, X., Clauer, C. R., R. Kunduri, B. S., Matzka, J., Stolle, C., and Weimer, D. R.: Geomagnetic response to solar wind dynamic pressure impulse events at high-latitude conjugate points, J. Geophys. Res., 118, 6055-6071, doi:10.1002/jgra.50555, 2013.
Kim, H., Clauer, C. R., Deshpande, K., Lessard, M. R., Weatherwax, A. T., Bust, G. S., Crowley, G., and Humphreys, T. E.: Ionospheric irregularities during a substorm event: Observations of ULF pulsations and GPS scintillations, J. Atmos. Solar-Terr. Phys., 114, 1-8, doi:10.1016/j.jastp.2014.03.006, 2014.

Kinrade, J., Mitchell, C. N., Yin, P., Smith, N., Jarvis, M. J., Maxfield, D. J., Rose, M. C., Bust, G. S., and Weatherwax, A. T.: Ionospheric scintillation over Antarctica during the storm of 5-6 April 2010, J. Geophys. Res., 117, A05304, doi:10.1029/2011JA017073, 2012.

Lessard, M. R., Weatherwax, A. T., Spasojevic, M., Inan, U., Gerrard, A., Lanzerotti, L., Ridley, A., Engebretson, M. J., Petit, N., Clauer, R., LaBelle, J., Mende, S., Frey, H., Pilipenko, S., Rosenberg, T. J., and Detrick, D.: PENGUIn multi-instrument observations of dayside high-latitude injections during the March 23, 2007 substorm, J. Geophys. Res., 114, A00C11, doi:10.1029/2008JA013507, 2009.

Lightsey, E. G., Humphreys, T. E., Bhatti, J. A., Joplin, A., O'Hanlon, B. W., and Powell, S.: Demonstration of a Space Capable Miniature Dual Frequency GNSS Receiver, Navigation, 61, 53-64, 2014.

Melville, R., Stillinger, A., Gerrard, A., and Weatherwax, A.: Sustanable energy at the 100-W level for scientific sites on the Antarctic Plateau: Lessons learned from the PENGUInAGO project, Rev. Sci. Instrum., Rev. Sci.Instrum, 85, 045117, doi:10.1063/1.4871555, 2014.

Mende, S. B., Harris, S. E., Frey, H. U., Angelopoulos, V., Russell, C. T., Donovan, E., Jackel, B., Greffen, M., and Peticolas, L. M.: The THEMIS array of ground-based observatories for the study of auroral substorms, Space Sci. Rev., 141, 357-387, 2008.

Musko, S., Clauer, C., Ridley, A. J., and Arnett, K. L.: Autonomous low-power magnetic data collection platform to enable remote high latitude array deployment, Rev. Sci. Instrum., 80, 044501, doi:10.1063/1.3108527, 2009.

O'Hanlon, B. W., Psiaki, M. L., Powell, S., Bhatti, J. A., Humphreys, T. E., Crowley, G., and Bust, G. S.: CASES: A smart, compact GPS software receiver for space weather monitoring, in: Proc 24th International Technical Meeting, Satellite Div., 1745-2753, Inst. of Navigation, Manassas, VA, 2011.

Prikryl, P., Spogli, L., Jayachandran, P. T., Kinrade, J., Mitchell, C. N., Ning, B., Li, G., Cilliers, P. J., Terkildsen, M., Danskin, D. W., Spanswick, E., Donovan, E., Weatherwax, A. T., Bristow, W. A., Alfonsi, L., De Franceschi, G., Romano, V., Ngwira, C. M., and Opperman, B. D. L.: Interhemispheric comparison of GPS phase scintillation at high latitudes during the magnetic-cloudinduced geomagnetic storm of 5-7 April 2010, Ann. Geophys., 29, 2287-2304, doi:10.5194/angeo-29-2287-2011, 2011.

Prikryl, P., Zhang, Y., Ebihara, Y., Ghoddousi-Fard, R., Jayachandran, P. T., Kinrade, J., Mitchell, C. N., Weatherwax, A. T., Bust, G., Cilliers, P. J., Spogli, L., Alfonsi, L., Romano, V., Ning, B., Li, G., Jarvis, M. J., Danskin, D. W., Spanswick, E., Donovan, E., and Terkildsen, M.: An interhemispheric comparison of GPS phase scintillation with auroral emission observed at the South Pole and from the DMSP satellite, Ann. Geophys., 56, 2037416X, 2013.

Warrington, E. M., Zaalov, N. Y., Naylor, J. S., and Stocker, A. J.: HF propagation modeling within the polar ionosphere, Radio Sci., 47, RSOL13, doi:10.1029/2011RS004909, 2012. 